\title{
Correction to: Pilot study of the multicentre DISCHARGE trial: image quality and protocol adherence results of computed tomography and invasive coronary angiography
}

Gianluca De Rubeis ${ }^{1}$ - Adriane E. Napp ${ }^{2} \cdot$ Peter Schlattmann $^{3} \cdot$ Jacob Geleijns ${ }^{4} \cdot$ Michael Laule $^{5} \cdot$ Henryk Dreger $^{5}$. Klaus Kofoed ${ }^{6,7}$. Mathias Sørgaard ${ }^{7}$. Thomas Engstrøm ${ }^{7}$. Hans Henrik Tilsted ${ }^{7}$. Alberto Boi ${ }^{8}$. Michele Porcu ${ }^{9}$. Stefano Cossa ${ }^{10}$. José F. Rodríguez-Palomares ${ }^{11}$. Filipa Xavier Valente ${ }^{11} \cdot$ Albert Roque $^{12} \cdot$ Gudrun Feuchtner $^{13}$. Fabian Plank ${ }^{14}$. Cyril Štěchovský ${ }^{15}$. Theodor Adla ${ }^{16}$. Stephen Schroeder ${ }^{17}$. Thomas Zelesny ${ }^{18}$. Matthias Gutberlet ${ }^{19}$ - Michael Woinke ${ }^{20}$ - Mihály Károlyi ${ }^{21}$. Júlia Karády ${ }^{22}$ • Patrick Donnelly ${ }^{22}$ - Peter Ball ${ }^{23}$. Jonathan Dermot Dodd ${ }^{24}$ - Mark Hensey ${ }^{25}$ - Massimo Mancone ${ }^{26}$. Andrea Ceccacci ${ }^{26}$ - Marina Berzina ${ }^{27}$. Ligita Zvaigzne ${ }^{28}$. Gintare Sakalyte ${ }^{29}$. Algidas Basevičius ${ }^{30}$. Małgorzata Ilnicka-Suckiel ${ }^{31}$ • Donata Kuśmierz ${ }^{32}$. Rita Faria ${ }^{33}$. Vasco Gama-Ribeiro ${ }^{33}$ - Imre Benedek ${ }^{34}$. Teodora Benedek ${ }^{34}$ • Filip Adjić ${ }^{35}$. Milenko Čanković ${ }^{36}$. Colin Berry ${ }^{37}$. Christian Delles ${ }^{37}$ - Erica Thwaite ${ }^{38}$ - Gershan Davis ${ }^{39}$ - Juhani Knuuti ${ }^{40}$ - Mikko Pietilä ${ }^{41}$. Cezary Kepka ${ }^{42}$ - Mariusz Kruk ${ }^{43} \cdot$ Radosav Vidakovic $^{44}$ - Aleksandar N. Neskovic ${ }^{44}$ - Iñigo Lecumberri ${ }^{45}$. Ignacio Diez Gonzales ${ }^{46} \cdot$ Balazs Ruzsics $^{47} \cdot$ Mike Fisher $^{47} \cdot$ Marc Dewey $^{2} \cdot$ Marco Francone $^{1}$ (D) - The DISCHARGE Trial Group

Published online: 24 April 2020

(C) European Society of Radiology 2020

Correction to: European Radiology (2020) 30:1997-2009 https://doi.org/10.1007/s00330-019-06522-z

The original version of this article, published on 16 December 2019, unfortunately contained two mistakes. Firstly, the name of Jonathan Dermot Dodd was presented incorrectly. Secondly, the information about the equal contribution of Gianluca De Rubeis and Adriane E. Napp, and Marc Dewey and Marco Francone is missing. The corrected author list is given above and the missing article note below. Furthermore, affiliation 48 from the original version of the article was a duplicate and is therefore removed.

Gianluca De Rubeis and Adriane E. Napp contributed equally to this work; Marc Dewey and Marco Francone contributed equally to this work.

The online version of the original article can be found at https://doi.org/ 10.1007/s00330-019-06522-Z

Marco Francone

marco.francone@uniroma1.it

Extended author information available on the last page of the article 


\section{Affiliations}

Gianluca De Rubeis ${ }^{1}$ - Adriane E. Napp ${ }^{2} \cdot$ Peter Schlattmann $^{3}$ • Jacob Geleijns ${ }^{4}$ • Michael Laule ${ }^{5} \cdot$ Henryk Dreger $^{5}$. Klaus Kofoed $^{6,7}$. Mathias Sørgaard ${ }^{7} \cdot$ Thomas Engstrøm $^{7} \cdot$ Hans Henrik Tilsted $^{7} \cdot$ Alberto Boi $^{8} \cdot$ Michele Porcu $^{9}$. Stefano Cossa ${ }^{10}$. José F. Rodríguez-Palomares ${ }^{11}$ • Filipa Xavier Valente ${ }^{11} \cdot$ Albert Roque $^{12} \cdot$ Gudrun Feuchtner $^{13}$. Fabian Plank ${ }^{14}$. Cyril Štěchovský ${ }^{15}$. Theodor Adla ${ }^{16}$. Stephen Schroeder ${ }^{17}$. Thomas Zelesny ${ }^{18}$. Matthias Gutberlet ${ }^{19}$ - Michael Woinke ${ }^{20}$ - Mihály Károlyi ${ }^{21}$ - Júlia Karády ${ }^{22}$ • Patrick Donnelly ${ }^{22}$ - Peter Ball ${ }^{23}$. Jonathan Dermot Dodd ${ }^{24}$ - Mark Hensey ${ }^{25}$ - Massimo Mancone ${ }^{26}$. Andrea Ceccacci ${ }^{26}$ - Marina Berzina ${ }^{27}$. Ligita Zvaigzne ${ }^{28}$. Gintare Sakalyte ${ }^{29}$. Algidas Basevičius ${ }^{30}$ • Małgorzata Ilnicka-Suckiel ${ }^{31}$ • Donata Kuśmierz ${ }^{32}$. Rita Faria ${ }^{33}$. Vasco Gama-Ribeiro ${ }^{33}$ - Imre Benedek ${ }^{34}$. Teodora Benedek ${ }^{34}$ • Filip Adjić ${ }^{35}$. Milenko Čanković ${ }^{36}$. Colin Berry ${ }^{37}$. Christian Delles ${ }^{37}$. Erica Thwaite ${ }^{38}$ - Gershan Davis ${ }^{39}$. Juhani Knuuti ${ }^{40}$ - Mikko Pietilä ${ }^{41}$. Cezary Kepka ${ }^{42}$. Mariusz Kruk ${ }^{43}$. Radosav Vidakovic ${ }^{44}$. Aleksandar N. Neskovic ${ }^{44}$ - Iñigo Lecumberri ${ }^{45}$. Ignacio Diez Gonzales ${ }^{46}$ - Balazs Ruzsics ${ }^{47}$ - Mike Fisher ${ }^{47} \cdot$ Marc Dewey $^{2} \cdot$ Marco Francone $^{1}$ (D) $\cdot$ The DISCHARGE Trial Group

1 Department of Radiology, Sapienza University of Rome, Viale Regina Elena 324, 00161 Rome, Italy

2 Department of Radiology, Charité, Universitätsmedizin Berlin, Charitéplatz 1, 10117 Berlin, Germany

3 Department of Statistics, Informatics and Data Science, Jena University Hospital, Jena, Germany

4 Department of Radiology, Leiden University Medical Center, Albinusdreef 2, 2333 ZA Leiden, The Netherlands

5 Department of Cardiology, Charité, Universitätsmedizin Berlin, Charitéplatz 1, 10117 Berlin, Germany

6 Department of Radiology, Rigshospitalet Region Hovedstaden, Rigshospitalet 9, 2100 Copenhagen, Denmark

7 Department of Cardiology, Rigshospitalet Region Hovedstaden, Rigshospitalet 9, 2100 Copenhagen, Denmark

8 Department of Cardiology, Azienda Ospedaliera Brotzu, Cagliari, CA, Italy

9 Department of Radiology, Azienda Ospedaliera Universitaria di Cagliari, AOU di Cagliari, Polo di Monserrato, 09042 Monserrato, CA, Italy

10 Department of Radiology, Azienda Ospedaliera Brotzu, Cagliari, CA, Italy

11 Department of Cardiology, Hospital Universitari Vall d'Hebron, Institut de Recerca (VHIR), Universitat Autònoma de Barcelona, Passeig de Vall d'Hebron 119, 08035 Barcelona, Spain

12 Department of Radiology, Hospital Universitari Vall d'Hebron, Institut de Recerca (VHIR), Universitat Autònoma de Barcelona, Passeig de Vall d'Hebron 119, 08035 Barcelona, Spain

13 Department of Radiology, Medical University Innsbruck, Anichstr. 35, 6020 Innsbruck, Austria

14 Department of Cardiology, Medical University Innsbruck, Anichstr. 35, 6020 Innsbruck, Austria

15 Department of Cardiology, University Hospital Motol, Vuvalu 84, 15006 Prague 5, Czech Republic
16 Department of Radiology, University Hospital Motol, Vuvalu 84, 15006 Prague 5, Czech Republic

17 Department of Cardiology, ALB FILS KLINIKEN GmbH, Eichertstrasse 3, 73035 Goeppingen, Germany

18 Department of Radiology, ALB FILS KLINIKEN GmbH, Eichertstrasse 3, 73035 Goeppingen, Germany

19 Department of Radiology, University of Leipzig Heart Centre, Strümpellstrasse 39, 04289 Leipzig, Germany

20 Department of Cardiology, University of Leipzig Heart Centre, Strümpellstrasse 39, 04289 Leipzig, Germany

21 MTA-SE Cardiovascular Imaging Center, Heart and Vascular Center, Semmelweis University, Varosmajor u 68, Budapest 1122, Hungary

22 Department of Cardiology, Southeastern Health and Social Care Trust, Upper Newtownards Road Ulster, Belfast BT16 1RH, UK

23 Department of Radiology, Southeastern Health and Social Care Trust, Upper Newtownards Road Ulster, Belfast BT16 1RH, UK

24 Department of Radiology, St. Vincent's University Hospital and National University of Ireland, Belfield Campus, 4, Dublin, Ireland

25 Department of Cardiology, St. Vincent's University Hospital, Belfield Campus, 4, Dublin, Ireland

26 Department of Cardiovascular, Respiratory, Nephrology, Anesthesiology and Geriatric Sciences, Sapienza University of Rome, Viale Regina Elena 324, 00161 Rome, Italy

27 Department of Cardiology, Paul Stradins Clinical University Hospital, Pilsoṇu Street 13, Riga 1002, Latvia

28 Department of Radiology, Paul Stradins Clinical University Hospital, Pilsoņu Street 13, Riga 1002, Latvia

29 Department of Cardiology, Lithuanian University of Health Sciences, Eivelniu 2, 50009 Kaunas, Lithuania

30 Department of Radiology, Lithuanian University of Health Sciences, Eivelniu 2, 50009 Kaunas, Lithuania 
31 Department of Cardiology, Wojewodzki Szpital Specjalistyczny We Wroclawiu, Ul. Henryka Michala Kamienskiego, 51-

124 Wroclaw, Poland

32 Department of Radiology, Wojewodzki Szpital Specjalistyczny We Wroclawiu, Ul. Henryka Michala Kamienskiego, 51-

124 Wroclaw, Poland

33 Department of Cardiology, Centro Hospitalar de Vila Nova de Gaia, Rua Conceicao Fernandes, 4434-502 Vila Nova de Gaia, Portugal

34 Department of Cardiology, Cardio Med Medical Center, 54015622 decembrie 1989, Targu-Mures, Romania

35 Radiology Department Imaging Center, Institute of Cardiovascular Diseases of Vojvodina, Put dr Goldmana 4, Sremska Kamenica, Novi Sad 212014, Serbia

36 Department of Cardiology, Institute of Cardiovascular Diseases of Vojvodina, Put dr Goldmana 4, Sremska Kamenica, Novi Sad 212014, Serbia

37 Institute of Cardiovascular and Medical Sciences, University of Glasgow, University Place 126, Glasgow G12 8TA, UK

38 Department of Radiology, Aintree University Hospital, Longmoor Lane, Liverpool L9 7AL, UK
39 Department of Cardiology, Aintree University Hospital, Longmoor Lane, Liverpool L9 7AL, UK

40 Turku PET Centre, Turku University Hospital and University of Turku, Kiinamyllynkatu 4-8, 20120 Turku, Finland

41 Heart Centre, Turku University Hospital, Kiinamyllynkatu 4-8, FI20120 Turku, Finland

42 Department of Radiology, The Institute of Cardiology in Warsaw, Ul. Alpejska 42, 04-628 Warsaw, Poland

43 Department of Cardiology, The Institute of Cardiology in Warsaw, Ul. Alpejska 42, 04-628 Warsaw, Poland

44 Department of Cardiology, Clinical Hospital Center Zemun, Vukova 9, Belgrade-Zemun 11080, Serbia

45 Department of Radiology, Basurto University Hospital, Avenida Montevideo 18, 48013 Bilbao, Spain

46 Department of Cardiology, Basurto University Hospital, Avenida Montevideo 18, 48013 Bilbao, Spain

47 Department of Cardiology, Royal Liverpool and Broadgreen University Hospitals, Prescot Street, Liverpool L7 8XP, UK 